\title{
Special section on modeling of reactive systems
}

\author{
Étienne Craye · Abdoulaye Gamatié
}

Published online: 17 May 2013

(C) Springer Science+Business Media New York 2013

This Special Section of J-DEDS on Modeling of Reactive Systems was inspired by the eighth edition of the French-speaking conference "Modélisation des Systèmes Réactifs-MSR 2011." MSR 2011 was organized by the LAGIS (Laboratoire d'Automatique, Génie Informatique et Signal) and LIFL (Laboratoire d'Informatique Fondamentale de Lille) laboratories, and held at École Centrale de Lille, France, on November 16-18, 2011. MSR is a conference that traditionally gathers French-speaking researchers from both the Computer Science and the Automatic Control communities, and that are involved in the modeling, analysis and control of reactive systems.

The articles presented at MSR 2011 covered a wide range of topics: simulation of discrete event systems, programming for discrete controller synthesis, analysis and control of timed and embedded systems, design of synchronous and multi-clock reactive systems. Following the MSR 2011 conference, the MSR Steering Committee invited a small number of authors who attended MSR 2011 to submit full journal papers for publication in a Special Section of J-DEDS on the topic of Modeling of Reactive Systems. These invitations reflected the variety of problems related to the study of discrete event systems discussed during MSR 2011. The submitted new papers have been fully reviewed according to the standard editorial process of J-DEDS under the coordination of the Guest Editors and of Department Editor Stéphane Lafortune. In the end, three papers were selected for inclusion in this Special Section.

The article presented by José Echeveste, Arshia Cont, Jean-Louis Giavitto and Florent Jacquemard describes an operational semantics of a domain-specific language, named Antescofo, for real-time musician-computer interaction. This work

É. Craye

Ecole Centrale de Lille, Cité Scientifique, 59650 Villeneuve d'Ascq, France e-mail: etienne.craye@ec-lille.fr

A. Gamatié $(\bowtie)$

CNRS/LIRMM, 161 rue Ada, 34095 Montpellier Cedex 5, France

e-mail: abdoulaye.gamatie@lirmm.fr 
is an original attempt to bridge the gap between computer-assisted music and the formal approach to reactive systems and languages. Antescofo is a process algebra whose semantics is provided through parametric timed automata. It formalizes the score of the musicians and how a computer system, responsible of the accompaniment, should react to the actual interpretation of the musicians. Indeed, the most important feature of the language is its ability to define several strategies to handle deviations, during real performance, from the score by the musicians.

The article proposed by Hervé Marchand, Gwenaël Delaval and Éric Rutten presents an integration of discrete controller synthesis (DCS) in a reactive programming language compiler. It promotes an easy access to DCS techniques for users in order to implement adaptive and reconfigurable. Moreover, it makes possible the programming of closed-loop adaptation controllers, which are useful to the flexible execution of functionalities with respect to changing resource and environment conditions. The resulting tool is concretely built upon the basis of a reactive programming language compiler, where the programming units describe behaviors that can be modeled in terms of transition systems. The compiler integrates this with a DCS tool, making it a new environment for formal methods. The authors define the trace semantics of the suggested language, describe its compilation and discuss the implementation with examples.

The last article from Olivier H. Roux, Didier Lime and Claude Martinez deals with shrinking of Time Petri nets. This study aims to relax the restriction induced by a pre-determined schedule that is assumed in dioid algebras-based approaches related to interval shrinking problem. The authors assume that the sequence of resources usage is not a priori determined. All possible trajectories can be considered and the control would disable those which would lead to constraints violation for a Time Petri net model of a plant. The existence of a control law that guarantees the temporal constraints are met is translated into the existence of a set of interval shrinkings on the Time Petri net, without modification of its structure. It is shown that the interval shrinking problem is decidable for CTL properties on bounded Time Petri nets. The presented result has been implemented in a prototype tool, named ROMEO and illustrated on a case study from the literature.

We would like to express our gratitude towards the authors for their contributions and timely revisions, to the reviewers for their work and constructive suggestions that contributed to the quality of the final articles, and also to the editorial board of J-DEDS for giving us the opportunity to present this selection of contributions. 